\title{
Relative Monocytosis in Patients with Erythrocytosis*
}

\author{
Hiroshi Fujita $^{1 \#}$, Akira Ohwada ${ }^{2}$, Naoko Handa ${ }^{2}$, Shigeko Nishimura ${ }^{1}$ \\ ${ }^{1}$ Department of Transfusion Medicine, Tokyo Metropolitan Bokutoh Hospital, Tokyo, Japan; ${ }^{2}$ Department of Internal Medicine, To- \\ kyo Metropolitan Bokutoh Hospital, Tokyo, Japan. \\ Email: \#yuketsuka@bokutoh-hp.metro.tokyo.jp
}

Received December $21^{\text {st }}, 2012$; revised January $22^{\text {nd }}, 2013$; accepted February $6^{\text {th }}, 2013$

\begin{abstract}
Polycythemia vera (PV) is characterized by leukocytosis, thrombocytosis, and low mean capsular volume (MCV). No specific hematological findings are observed in patients with erythrocytosis of other origins. However, recombinant erythropoietin (EPO) increases the number of mononuclear cells in the peripheral blood. Therefore, we hematologically examined monocytes in patients with erythrocytosis. The medical records of 48 erythrocytosis patients (hemoglobin: men, >18.5 g/dL; women, >16.5 g/dL) collected between August 2005 and July 2012 were reviewed for age, gender, hematology, and serum erythropoietin level. Patients were divided into 3 groups: PV $(\mathrm{N}=17)$, reactive erythrocytosis (RE, $\mathrm{N}=25$ ), and EPO-elevated erythrocytosis (EPO-E, $\mathrm{N}=6$ ). We compared monocytes in the hemogram among the 3 groups and examined the correlation between hematological data and serum EPO level. The percent monocytes in the hemogram were $3.4 \% \pm 0.3 \%, 7.4 \% \pm 0.4 \%$, and $12.3 \% \pm 1.1 \%$ for the PV, RE, and EPO-E groups, respectively, which were significant $(\mathrm{p}<0.05)$. Absolute monocyte counts in the EPO-E group $\left(1.0 \pm 0.1 \times 10^{9} / \mathrm{L}\right)$ were significantly higher than those in the PV $\left(0.6 \pm 0.1 \times 10^{9} / \mathrm{L}\right)$ and $\mathrm{RE}\left(0.5 \pm 0.3 \times 10^{9} / \mathrm{L}\right)$ groups. There were $2(12 \%), 0$, and $3(50 \%)$ patients with monocytosis $\left(>1.0 \times 10^{9} / \mathrm{L}\right)$ in the PV, RE, and EPO-E groups, respectively. A significant correlation was observed between percent monocyte or absolute monocyte count and serum EPO level. In conclusion, monocyte count in hemograms may be useful for diagnosing EPO-elevated erythrocytosis.
\end{abstract}

Keywords: Erythropoietin; Monocyte; Erythrocytosis

\section{Introduction}

Erythrocytosis is identified based on hemoglobin (Hb) levels using hematology assays (Hb: men, $>18.5 \mathrm{~g} / \mathrm{dL}$; women, $>16.5 \mathrm{~g} / \mathrm{dL}$ ). The causes of erythrocytosis include various pathological diseases. Among the types of erythrocytosis, polycythemia vera (PV) is classified as a myeloproliferative neoplasm [1]. PV is characterized by leukocytosis, thrombocytosis, and low to normal serum levels of erythropoietin in clinical tests [1,2]. We previously examined the laboratory values between PV and reactive erythrocytosis [3-6]. First, we reported that microcytosis may be a characteristic of the PV clinical course and a good marker for the presence of the JAK2 V617F mutation [3]. Second, the activities of coagulation factors were significantly lower in PV patients than in reactive erythrocytosis patients [4]. Serum cholesterol and G-CSF levels in PV patients were lower than that in those with reactive erythrocytosis $[5,6]$.

Recombinant erythropoietin increases the number of mononuclear cells in the peripheral blood [7,8]. These

\footnotetext{
${ }^{*}$ The authors have no conflicts of interest to report.

The authors alone are responsible for the content and writing of this article.

\#Corresponding author.
}

cells are used as endothelial progenitor cells in regeneration medicine. There have been no previous reports on the monocyte count in erythrocytosis.

First, we compared hematological findings among the 3 groups: PV, reactive erythrocytosis (RE), and EPOelevated erythrocytosis (EPO-E). Monocyte count in EPO-E patients was higher than that in PV or RE patients. Next, we examined the correlation between monocytes and erythropoietin using a hemogram. Serum levels of erythropoietin were positively correlated to the relative monocyte and absolute monocyte counts.

Therefore, we concluded that relative monocytosis in hematology may be a useful parameter for diagnosing EPO-E.

\section{Patients and Methods}

\subsection{Patients}

Tokyo Metropolitan Bokutoh Hospital is located in Eastern Tokyo. We retrospectively reviewed the medical records of 48 patients with erythrocytosis (hemoglobin [Hb]: men, >18.5 g/dL; women, >16.5 g/dL) collected between August 2005 and July 2012. Medical records were reviewed for age, gender, and laboratory test results. 
Venous blood was collected into evacuated tubes to measure hematology and serum erythropoietin for the initial outpatients in our departments. Patients were divided into 3 groups according to the new World Health Organization (WHO) classification as follows: PV group $(\mathrm{N}=17)$, reactive erythrocytosis group (RE, $\mathrm{N}=25)$, and EPO elevated erythrocytosis (EPO-E, $\mathrm{N}=6$; serum EPO level, >29 mIU/L). The RE group could not be diagnosed with PV despite erythrocytosis (serum erythropoietin within normal limits); erythrocytosis improved due to phlebotomies. The RE group revealed neither JAK2 V617F mutation nor hypoxia. Furthermore, patients with EPO-E exhibited erythropoietin-producing tumors (renal cell carcinoma, $\mathrm{N}=3$ ) and obesity with hypoxia (pickwickian syndrome, $\mathrm{N}=3$ ). The study design was approved by the ethics review board of our institution.

\subsection{Statistical Analysis}

We compared the differences among the three erythrocytosis groups by using Wilcoxon's analysis. Data are expressed as group means \pm standard errors of the mean or medians with interquartile ranges. Rates of monocytosis were compared by using the chi-square test with Yates's correction. All statistical calculations were performed using JMP version 8.0 software (SAS Institute, Inc., Cary, NC), and significance was defined as p < 0.05 .

The correlation between the serum erythropoietin level and hematology results was assessed by using linear regression analysis. Significance for the linear regression analysis results was defined as $\mathrm{p}<0.0001$.

\section{Results}

\subsection{Hematology in Patients with Erythrocytosis}

Of the 48 patients with erythrocytosis, 17 were diagnosed with PV. The remaining 31 patients with erythrocytosis tested negative for the JAK2 V617F mutation. The 31 patients were divided into 2 groups, including a reactive erythrocytosis group (RE; $\mathrm{N}=25$ ) and an erythropoietin elevated erythrocytosis group $(>29 \mathrm{mIU} / \mathrm{L}, \mathrm{EPO}-\mathrm{E} ; \mathrm{N}=$ $6)$.

Patients with PV were significantly older than those with RE and EPO-E; however, no gender differences were noted among the 3 groups.

Hematological tests performed upon initial presentation revealed that the numbers of erythrocytes, leukocytes, and platelets were significantly higher in the PV group than in the RE and EPO-E groups (Table 1). However, percent monocytes according to the hemogram were $3.4 \% \pm 0.3 \%, 7.4 \% \pm 0.4 \%$, and $12.3 \% \pm 1.1 \%$, in the PV, RE, and EPO-E groups, respectively, which were significant $(\mathrm{p}<0.05$, Table 1). Additionally, absolute monocyte counts were significantly higher in the EPO-E group than those in the PV and RE groups $(\mathrm{p}<0.05)$ (Table 1).

The rate of monocytosis $\left(>1.0 \times 10^{9} / \mathrm{L}\right)$ was significantly higher in EPO-E patients $(50 \%$; $3 / 6)$ than in RE patients $(0 \%, 0 / 25)$, but not in PV patients $(12 \%, 2 / 17)$ (Table 1).

\subsection{Relationship between Serum Erythropoietin Level and Hematological Findings in Erythrocytosis Patients}

Post-treatment for EPO-E group, including nephrectomy for renal cell carcinoma or weight control for obesity, resulted in a reduction in percent monocyte $(7.0 \% \pm$ $0.3 \%, \mathrm{p}<0.05$ vs. pre-treatment) and absolute monocyte counts $\left(0.5 \pm 0.03 \times 10^{9} / \mathrm{L}\right.$, not significant vs. pre-treatment). Serum levels of erythropoietin after treatment were significantly reduced compared with those before treatment (pre-treatment erythropoietin concentration; 115 $\pm 33 \mathrm{mIU} / \mathrm{L}$, post-treatment erythropoietin concentration; $25 \pm 4 \mathrm{mIU} / \mathrm{L}, \mathrm{p}<0.05)$.

Among hematology parameters (hemoglobin, red cell counts, hematocrit, platelet counts, leukocyte counts, absolute monocyte counts and percent monocytes), only percent monocytes showed significant positive correlations with the serum erythropoietin level $\left(r^{2}=0.41111\right.$, $\mathrm{p}$ $<0.0001)$. There was no significant relationship between erythrocytosis and the serum erythropoietin levels (RBC: $r^{2}=0.016192$, not significant, hemoglobin: $r^{2}=0.000795$, not significant, hematocrit: $r^{2}=0.061684$, not significant).

\section{Discussion}

We previously reported that the serum levels of total cholesterol and granulocyte colony stimulating factor (G-CSF), and the activities of some clotting factors are lower in patients with PV than in those with non-PV erythrocytosis [4-6]. Microcytosis in hematology was lower in the PV group than in the RE and EPO-E groups (Table 1). These findings are similar to the results of our previous report [3]. Erythrocytosis diagnosis was performed by general clinical laboratories, such as hematology and biochemistry laboratories. We speculated that monocyte number in hematology may be a predictor for elevated serum erythropoietin levels because recombinant erythropoietin increased the number of mononuclear cells in the peripheral blood $[7,8]$.

Erythropoietin receptors are present on the membranes of monocytes [9], and monocytes in the bone marrow are mobilized by erythropoietin to the peripheral blood $[7,8]$. Erythropoietin induces monocyte chemoattractant protein-1 (MCP-1) in the endothelial cells [10]. Therefore, we speculated that erythropoietin may be directly or indirectly effective for mobilizing monocytes through 
Table 1. Clinical profiles and lipid profiles of patients with erythrocytosis.

\begin{tabular}{|c|c|c|c|}
\hline Clinical data & $P V$ group $(\mathrm{N}=17)$ & $R E$ group $(\mathrm{N}=25)$ & EPO-E group $(\mathrm{N}=6)$ \\
\hline Age & $69(2)^{*}$ & $54(2)^{*}$ & $59(5)^{*}$ \\
\hline Gender $(\mathrm{M} / \mathrm{F})$ & $6 / 8$ & $\begin{array}{c}54(2) \\
21 / 1\end{array}$ & $5 / 1$ \\
\hline Underlying diseases and conditions & & & $\begin{array}{c}\text { Renal cell carcinoma (3) } \\
\text { Obesity (3) }\end{array}$ \\
\hline \multicolumn{4}{|l|}{ Hematology } \\
\hline Hemoglobin (g/dL) & $18.8(0.4)$ & $19.2(0.2)$ & $18.8(0.4)$ \\
\hline Hematocrit (\%) & $57.8(0.9)$ & $56.2(0.7)$ & $58.3(1.7)$ \\
\hline Red blood cells $\left(\times 10^{9} / \mathrm{L}\right)$ & $6997(220)$ & $5870(60)^{*}$ & $6700(30.0)^{*}$ \\
\hline MCV (fL) & $84(3)$ & $96(1)$ & $88(4)$ \\
\hline Platelets $\left(\times 10^{9} / \mathrm{L}\right)$ & $401.9(43.1)$ & $198.0(9.0)^{*}$ & $181.0(3.0)^{*}$ \\
\hline Leukocytes $\left(\times 10^{9} / \mathrm{L}\right)$ & $16.3(2.3)$ & $6.8(0.3)^{*}$ & $7.7(1.1)^{*}$ \\
\hline \% Monocyte & $3.4(0.3)^{* *}$ & $7.4(0.4)^{* *}$ & $12.3(1.1)$ \\
\hline \% Monocyte + lymphocyte & $13.8(1.2)$ & $37.7(1.9)$ & $33.8(3.2)$ \\
\hline Absolute monocyte $\left(\times 10^{9} / \mathrm{L}\right)$ & $0.6(0.1)^{* *}$ & $0.5(0.3)^{* * *}$ & $1.0(0.1)$ \\
\hline Absolute monocyte + Lymphocyte $\left(\times 10^{9} / \mathrm{L}\right)$ & $2.0(0.2)$ & $2.4(0.1)$ & $2.5(0.4)$ \\
\hline Absolute monocyte $>1.0 \times 10^{9} / \mathrm{L}$ & $2,12 \%$ & $0,0 \%{ }^{* *}$ & $3,50 \%$ \\
\hline Serum erythropoietin (mIU/L) & $9.1(0.8)^{* *}$ & $16.1(1.4)^{* *}$ & 115 (33) \\
\hline
\end{tabular}

Data represent the means with standard errors in parentheses. ${ }^{*} \mathrm{p}<0.05 \mathrm{vs}$. PV, ${ }^{* *} \mathrm{p}<0.05 \mathrm{vs}$. EPO-E.

MCP-1. However, some types of renal cell carcinoma produce macrophage colony stimulating factor (M-CSF) and granulocyte-macrophage colony-stimulating factor (GM-CSF) [11]. We hypothesized that relative monocytosis was due to the direct effect of erythropoietin and other effects of GM-CSF and M-CSF produced by renal cell carcinoma. Because few reports are available regarding the effects of serum GM-CSF and M-CSF in obese individuals, we hypothesized that relative monocytosis in EPO-E patients may be due to elevated erythropoietin, but not due to elevated GM-CSF and M-CSF. However, we did not measure serum levels of GM-CSF and M-CSF in all subjects.

The role of monocytes in erythrocytosis is unclear. Several reports showed that monocytes suppress erythropoiesis through the production of interferon gamma $[12,13]$. Therefore, we speculated that increase in the monocyte number in the peripheral blood may be due to negative feedback against excessive erythrocytosis. However, other mechanisms affect hematology hemograms in $\mathrm{PV}$, such as leukocytosis and neutrophilia [2].

In conclusion, to our knowledge, this is the first report of a positive correlation between serum erythropoietin level and the number of monocytes in the peripheral blood in patients with erythrocytosis. Relative monocytosis conducted in routine hematology assays may be due to elevated erythropoietin.

A limitation of this study is its small sample size, as the number of patients with erythrocytosis in a single metropolitan hospital is small. In this research, we did not examine the relationship between the monocyte count and the serum erythropoietin level in the non-erythyth- rocytic subjects.

\section{REFERENCES}

[1] J. W. Vardiman, J. Thiele, D. A. Arber, R. D. Brunning, M. J. Borowitz, A. Porwit, N. L. Harris, M. M. Le Beau, E. Hellstrom-Lindberg, A. Tefferi and C. D. Bloomfield, "The 2008 Revision of the World Health Organization (WHO) Classification of Myeloid Neoplasms and Acute Leukemia: Rationale and Important Changes,” Blood, Vol. 114, No. 5, 2009, pp. 937-951. doi:10.1182/blood-2009-03-209262

[2] T. Barbui, A. Carobbio, A. Rambaldi and G. Finazzi, "Perspectives on Thrombosis in Essential Thrombocythemia and Polycythemia Vera: Is Leukocytosis a Causative Factor?” Blood, Vol. 114, No. 4, 2009, pp. 759-763.

[3] H. Fujita, S. Kurosawa, S. Nishimura, J. Tomiyama, T. Hamaki and A. Ohwada, "Significance of Microcytosis in the Clinical Course of Erythrocytosis,” Internet Journal of Geriatrics and Gerontology, Vol. 6, No. 1, 2010, pp. 14. doi:10.5580/e0b

[4] H. Fujita, R. Sakuma, J. Tomiyama, T. Hamaki, A. Ohwada, and S. Nishimura, "Relationship between Clotting Activity and Phosphatidylserine Expression on Erythrocyte Membranes in Polycythemia Vera Patients with the JAK2 V617F Mutation," Archives of Physiology Biochemistry, Vol. 117, No. 4, 2011, pp. 231-235. doi:10.3109/13813455.2011.571262

[5] H. Fujita, T. Hamaki, N. Handa, A. Ohwada, J. Tomiyama and S. Nishimura, "Hypocholesterolemia in Patients with Polycythemia Vera," Journal of Clinical Experimental Hematopathology, Vol. 52, No. 2, 2012, pp. 8589. doi:10.3960/jslrt.52.85

[6] H. Fujita, T. Hamaki, A. Ohwada, J. Tomiyama and S. Nishimura, "Serum Levels of Granulocyte Colony-stimulating Factor in JAK2 V617F-Positive vs. Negative Erythrocytosis,” International Journal of Laboratory and He- 
matology, Vol. 33, No. 6, 2011, pp. e20-e21. doi:10.1111/j.1751-553X.2011.01336.X

[7] C. Heeschen, A. Aicher, R. Lehmann, M. Vasa, C. Urbich, C. Mildner-Rihm, H. Martin, A. M. Zeiher and S. Dimmeler, "Erythropoietin Is a Potent Physiologic Stimulus for Endothelial Progenitor Cell Mobilization,” Blood, Vol. 102, No. 4, 2004, pp. 1340-1346. doi:10.1182/blood-2003-01-0223

[8] A. V. R. Santhanam, L. V. d'Uscio, T. E. Peterson and Z. S. Katuzic, "Activation of Endothelial Nitric Oxide Synthase Is Critical for Erythropoietin-Induced Mobilization of Progenitor Cells,” Peptides, Vol. 29, No. 8, 2008, pp. 1451-1455. doi:10.1016/j.peptides.2008.03.016

[9] K. A. Lisowska, A. Debska-Slizien, E. Bryl, B. Rutkowski and J. M. Witkowski, "Erythropoietin Receptor is Expressed on Human Peripheral Blood T and B Lymphocytes and Monocytes and Is Modulated by Recombinant Human Erythropoietin Treatment," Artificial Organs, Vol. 34, No. 8, 2010, pp. 654-662.

[10] A. Desai, Y. Zhao, H. A. Lankford and J. S. Warren, "Ni- tric Oxide Suppresses EPO-Induced Monocyte Chemoattractant Protein-1 in Endothelial Cells: Implications for Atherogenesis in Chronic Renal Disease,” Laboratory Investigation, Vol. 86, No. 4, 2006, pp. 369-379. doi:10.1038/labinvest.3700396

[11] C. D. Gerharz, P. Reinecke, E. M. Schneider, M. Schmitz and H. E. Gabbert, "Secretion of GM-CSF and M-CSF by Human Renal Cell Carcinomas of Different Histologic Types,” Urology, Vol. 58, No. 5, 2001, pp. 821-827. doi:10.1016/S0090-4295(01)01371-1

[12] S. W. Mamus, S. Beck-Schroeder and E. D. Zanjani, "Suppression of Normal Human Erythropoiesis by Gamma Interferon in Vitro,” The Journal of Clinical Investigation, Vol. 75, No. 5, 1985, pp. 1496-1503. doi:10.1172/JCI111853

[13] J. J. Rinehart, E. D. Zanjani, B. Nomdedeu and B. J. Gormus, "Cell-Cell Interaction in Erythropoiesis," The Journal of Clinical Investigation, Vol. 62, No. 11, 1978, pp. 979-986. doi:10.1172/JCI109227 